\title{
PRIMER REGISTRO DE LABICHTHYS YANOI (MEAD \& RUBINOFF, 1966) (ACTINOPTERYGII, ANGUILLIFORMES) EN AGUAS URUGUAYAS
}

\author{
Marcos Nieddu \\ Área Ciencias del Mar, Instituto de Investigaciones Pesqueras "Prof. Dr. Víctor H. Bertullo", Facultad de \\ Veterinaria, Universidad de la República, Montevideo, Uruguay. \\ nieddupes@fvet.edu.uy
}

\section{RESUMEN}

Se cita por primera vez para Uruguay al pez nemíctido de aguas marinas profundas Labichthys yanoi (Mead \& Rubinoff, 1966) (Nemichthyidae), sobre la base de dos ejemplares capturados incidentalmente en la pesquería comercial con nasas para merluza negra (Dissostichus eleginoides Smitt, 1898) a una profundidad de $2212 \mathrm{~m}$ y $3000 \mathrm{~m}$. Esta especie es diferenciada de otros nemíctidos por poseer cuerpo alargado sin filamento caudal, línea lateral con un poro tubular por segmento, poros de la cabeza grandes, crestas sensitivas cortas por detrás del ojo y el orificio nasal anterior claro. Se registra la especie para Uruguay y se confirma su presencia en la región.

Palabras claves: Nemichthyidae, Uruguay, aguas profundas, captura incidental.

\section{ABSTRACT \\ First record of Labichthys yanoi (Mead \& Rubinoff, 1966) (Actinopterygii, Anguilliformes) in Uruguayan waters. The deep-water snipe eel Labichthys yanoi (Mead \& Rubinoff, 1966) (Nemichthyidae) is cited for Uruguay for first time, based on two specimens collected as by-catch of the commercial pot fishery for Patagonian Toothfish (Dissostichus eleginoides Smitt,1898), at $2212 \mathrm{~m}$ and $3000 \mathrm{~m}$ depth. This individuals are distinguished from other nemichthyids by having elongated body with no caudal filament, lateral line with a tubular pore per segment, large head pores, sensory short ridges behind the eye and light anterior nostril-tube. The species is recorded for Uruguay and its presence is confirmed for the region.}

Key words: Nemichthyidae, Uruguay, deep waters, by-catch.

\section{INTRODUCCIÓN}

El Orden Anguilliformes contiene ocho subórdenes compuestos por diecinueve familias de peces (Nelson, 2016). La familia Nemichthyidae perteneciente a este Orden y al Suborden Anguilloidei contiene tres géneros que presentan hábitos meso y batipelágicos: Labichthys Gill \& Ryder, 1883, Avocettina Jordan \& Davis, 1892 y Nemichthys Richardson, 1848 (Nielsen et al., 1978; Nelson, 2016). Los tres géneros y algunas de sus especies han sido citados en el Océano Atlántico Suroccidental (Menni et al., 1984; Figueroa et al., 1998; Vaz Ferreira et al., 1999; Nion et al., 2002; Costa et al., 2007; Cousseau, 2010; Melo et al., 2009) y en Uruguay se registró Nemichthys scolopaceus Richardson 1848 (Nion et al., 2002).

El presente estudio describe al pez óseo anguiliforme Labichthys yanoi (Mead \& Rubinoff, 1966) y lo cita por primera vez para aguas territoriales uruguayas.

\section{MATERIALES Y MÉTODOS}

Dos ejemplares completos de Labichthys yanoi fueron capturados incidentalmente en los meses de noviembre y diciembre de los años 2005 y 2006 por el B/P Viking Sky dirigido a la pesca de Merluza negra (Dissostichus eleginoides). Luego de colectados, fueron congelados a bordo en cámara de frío a $-30^{\circ} \mathrm{C}$. En tierra fueron descongelados, fotografiados, fijados en alcohol $70^{\circ}$ y depositados en la Colección de peces del Área Ciencias del Mar, del Instituto de Investigaciones Pesqueras (Facultad de Veterinaria, Universidad de la República, Montevideo, Uruguay) con el registro Anem/04 y Anem/05. Actualmente pertenecen a la Colección de peces del Museo de Historia Natural de Montevideo con los registros MHNM 3867 y MHNM 3868.

La pesquería donde fueron obtenidos los ejemplares operó con nasas en líneas a profundidades de 2500 m y más. Las líneas tienen entre 125 y 150 nasas con una distancia de $46 \mathrm{~m}$ entre ellas, dando un largo total de entre $5500 \mathrm{~m}$ y $6900 \mathrm{~m}$. Las nasas son trampas con forma cónica truncada, con un armazón de hierro y tienen un diámetro mayor de $140 \mathrm{~cm}$, un diámetro menor de $80 \mathrm{~cm}$ y una altura de $75 \mathrm{~cm}$, forradas de una malla de ca. $5 \mathrm{~cm}$. La carnada usada en la marea fue de Sardina canadiense congelada (Sardinops sagax) y lacha (Brevoortia aurea) según etiquetado.

Los caracteres merísticos y morfométricos fueron tomados según Nielsen \& Smith (1978). Debido generalmente a la pérdida de la porción caudal, estos autores proponen una longitud estándar artificial para la Familia que toma en cuenta la referencia de que 
para cada poro de la línea lateral por segmento corporal se corresponde su vértebra homóloga en la columna vertebral. Por tal motivo, se cuentan los primeros 100 poros de la línea lateral y en base a esto se estima el largo estándar a través de conteos de radios de aleta dorsal y anal. Para Labichthys, el largo estándar a la vértebra $n^{\circ} 100$ se expresa como SL100 y siendo D 100 el número de radios de la aleta dorsal a la vértebra 100 y A 100 los de la aleta anal a esta misma vértebra. Los radios de las aletas, poros y demás estructuras anatómicas fueron contados bajo lupa de mano Coil 2.3x/5.2d y lupa Nikon bifocal SMZ 2T, y los caracteres morfométricos fueron tomados con ictiómetro y calibre de mano Tajima Dial 15. La masa fue medida en una balanza Startorius Universal con precisión de 0,01 g.

\section{Abreviaturas referidas en el texto:}

MHNM: Museo de Historia Natural de Montevideo
ZCPAU: Zona Común de pesca ArgentinoUruguaya.

DINARA: Dirección Nacional de Recursos

Acuáticos.

UGEPA: Unidad de Gestión Pesquera Atlántica, DINARA.

IIP: Instituto de Investigaciones Pesqueras "Prof.

Dr. Víctor H. Bertullo".

B/l: Buque de Investigación.

$\mathrm{B} / \mathrm{P}$ : Buque pesquero comercial.

\section{Material examinado:}

1) MHNM 3867 (Figuras 1 y 2, Tabla 1), $395 \mathrm{~mm}$ de largo total, 3.58 g., 37050' S y 53010'W, 2212m, B/P "Viking Sky", Nov-Dic 2005. Colector: Marcos Nieddu.

2) MHNM 3868 (Figuras 1 y 3, Tabla 1), $670 \mathrm{~mm}$ de largo total, 29.17 g., 37020' S y 54⒉' W, 3000 m, B/P "Viking Sky", Nov-Dic 2006. Colector: Alejandro Perretta.

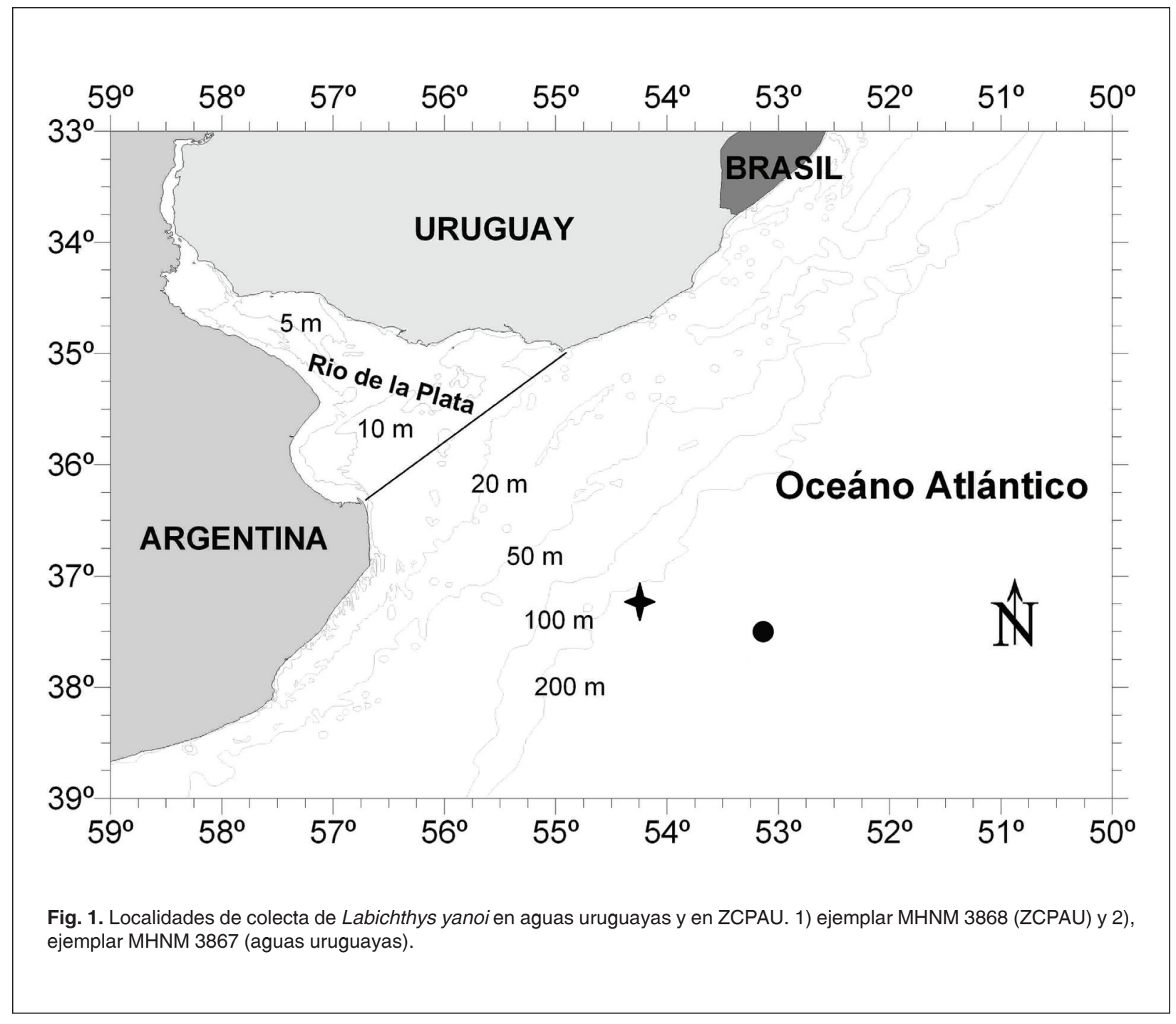

Bol. Soc. Zool. Uruguay (2a época). 2017. Vol. 26 (1): 9-15 ISSN: 0255-4402 


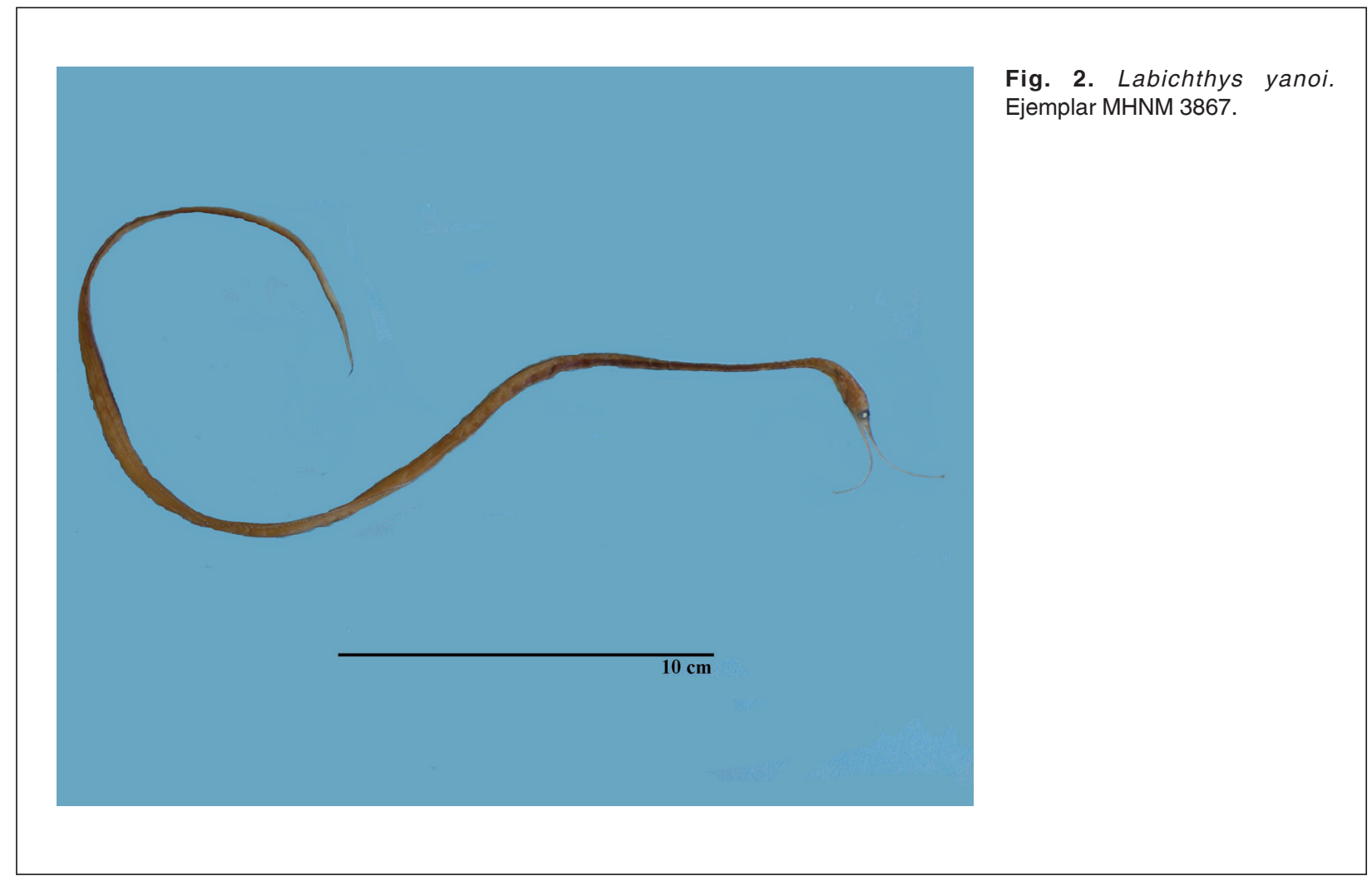

Fig. 3. Labichthys yanoi. Ejemplar MHNM 3868.

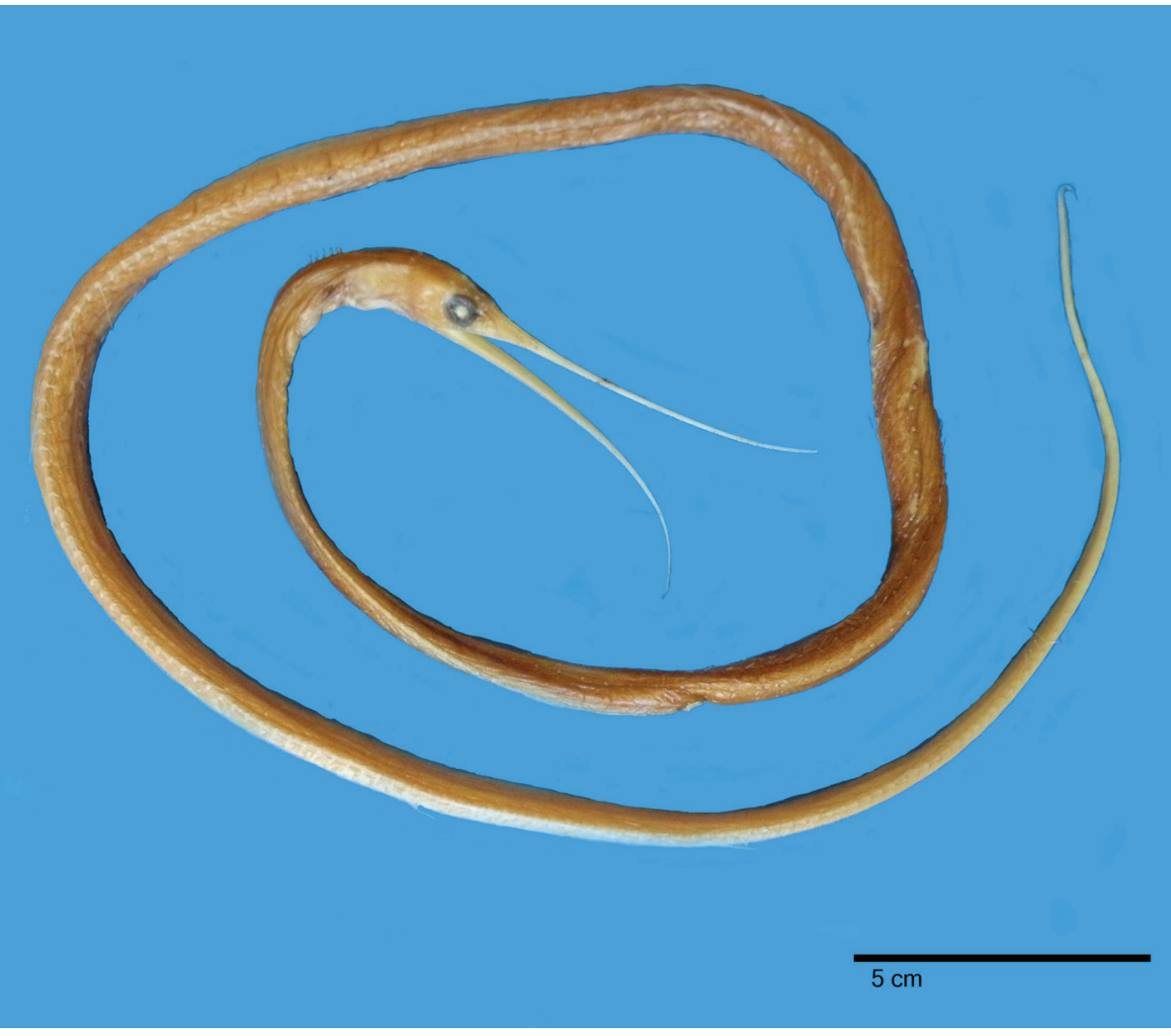

Bol. Soc. Zool. Uruguay (2ª época). 2017. Vol. 26 (1): 9-15 ISSN: 0255-4402 


\section{RESULTADOS Y DISCUSIÓN}

De acuerdo a Robins (1989) los especímenes analizados corresponden a la Familia Nemichthyidae. Los caracteres diagnósticos (merísticos y morfométricos) para Labichthys yanoi propuestos por Nielsen \& Smith (1978) son consistentes con los ejemplares examinados y se detallan a continuación.

Al igual que todos los nemíctidos el macho maduro se caracteriza por la pérdida de los dientes y el acortamiento de las mandíbulas, en las hembras maduras también hay reducción de dientes. Los individuos de Labichthys yanoi analizados poseen: 1) cuerpo alargado de sección plana con cola sin filamento caudal; 2) el comienzo de la aleta dorsal bien enfrentado a la base de las pectorales; 3) la mandíbula superior más larga que la inferior que termina en una protuberancia espatular con múltiples dientes con cúspide en dirección caudal (en ambas mandíbulas); 4) el sistema de la línea lateral está compuesto por una hilera simple de poros tubulares, observándose un poro por segmento que corresponde a cada vértebra de la columna vertebral; 5) los poros correspondientes a la cabeza son particularmente grandes; 6 ) el conducto nasal anterior es de color claro y tubular; 7) el diámetro del ojo está comprendido entre 21 y $34 \%$ del largo post orbital y presenta crestas sensitivas cortas por detrás; y 8) la posición del ano por debajo y apenas a continuación de las aletas pectorales.

El rango de largo total máximo registrado en Labichthys yanoi fue de $795 \mathrm{~mm}$ y el mínimo de 330 $\mathrm{mm}$, quedando los ejemplares comprendidos dentro de las tallas conocidas. Los datos merísticos y morfométricos presentados en la tabla de los ejemplares estudiados se encuentran dentro de los parámetros presentados por Mead \& Rubinoff (1966), aunque el porcentaje de la longitud preanal sobre la longitud a la vértebra no 100 Ls 100 se encuentra por debajo del rango propuesto (aunque en su trabajo el holotipo tampoco se encuentra contemplado dentro de los rangos de su muestra analizada).

Los nemíctidos son especies de hábitos meso y batipelágicas y se distribuyen en los océanos de todo el mundo. A nivel global Labichthys yanoi es encontrado en todo el hemisferio sur y ha sido capturado desde la superficie hasta profundidades de 2000 metros y más (Nielsen et al., 1978). Los registros más próximos a aguas uruguayas corresponden a ejemplares capturados en aguas argentinas frente a la Provincia de Buenos Aires (3820'S, 543' W, 0-1040 m de profundidad) (Nielsen

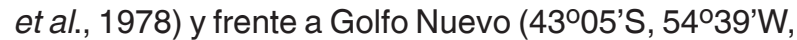
a $1000 \mathrm{~m}$ de profundidad) (Figueroa et al., 1998) (Figura 4).

Los cruceros que han operado en la ZCPAU, a pesar de que no todos estaban dirigidos a campañas de profundidad, obtuvieron muestras de peces mesopelágicos principalmente entre 200 y 1000 metros, e incluyeron los buques de investigación "Dana" y "Walther Herwig" (década de 1960 y 1970), el B/I "Shinkai Maru" (década del 70), los B/I "Evrika", "Capitán Oca Balda", "Dr. Eduardo Holmberg", "Kaiyo Maru", "Meteor" (década del 80 y década del 90) (Cousseau et al., 2012). También algunos buques pesqueros han obtenido muestras de peces de profundidad como el B/P "Cazon", el B/P "Lerez", el B/ P "Santa María Magdalena" ( Vaz Ferreira et al, 1999) y como en el caso del presente trabajo.

Para llevar a cabo estudios en ecología, biología evolutiva, biogeografía y conservación de un ecosistema, es menester conocer la composición de la biota y necesario para su monitoreo (Eastman, 2005). Sin embargo, las aguas profundas de la ZCPAU precisan de una mayor investigación que generará nuevos registros ictiológicos (Nion et al., 2002). La colecta y determinación de especies de peces de profundidad ha sido relativamente escasa en aguas uruguayas. Entre otras causas y según Nion et al.

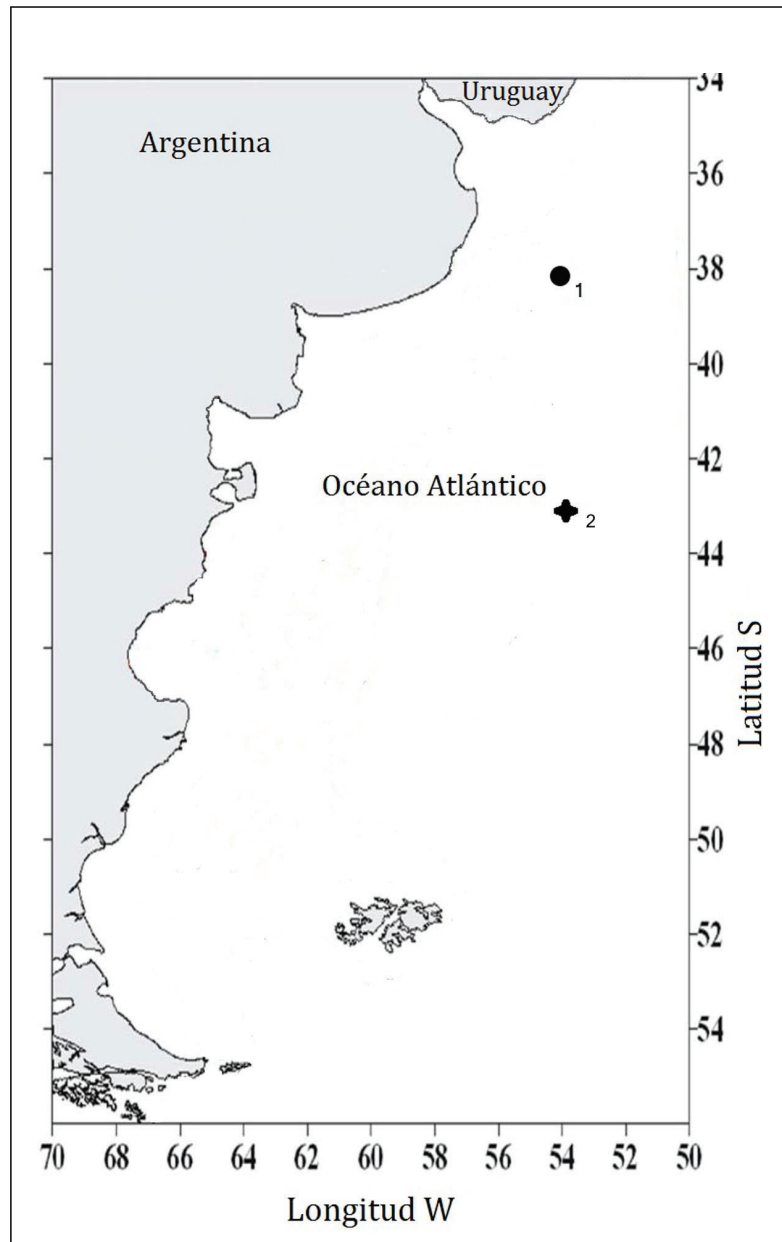

Fig. 4. Registros de ejemplares capturados frente a costas argentinas. 1) el ejemplar capturado por el B/I "Walter Herwig" en el año 1971 y 2) el ejemplar capturado por el B/I "Kaiyo Maru" en el año 1989. 


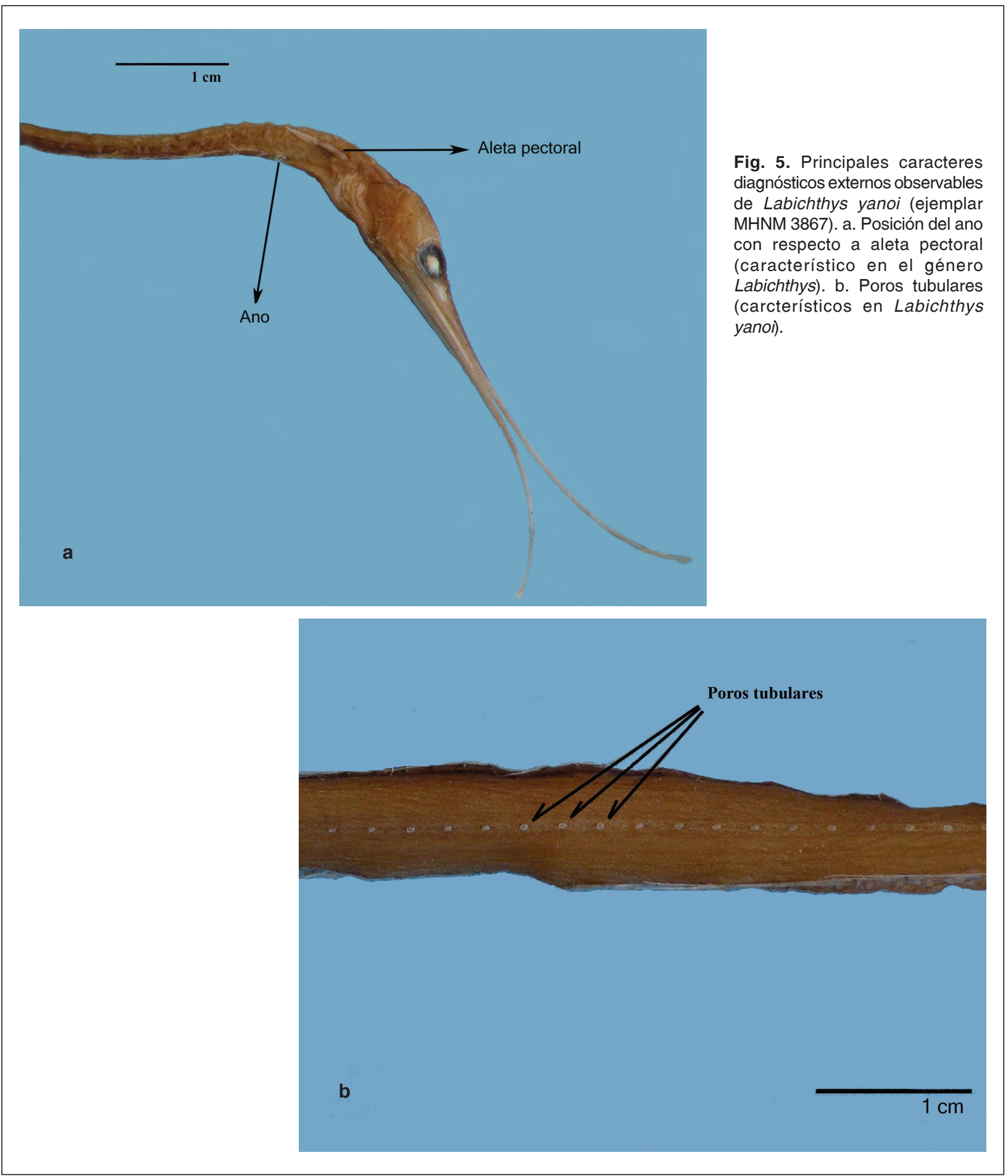

(2002), esto es debido a la falta de campañas de exploración de este hábitat tan particular).

\section{AGRADECIMIENTOS}

A la Dra. Graciela Fabiano y a la Dra. Cristina Aycaguer del Área Ciencias del Mar del IIP de la
Facultad de Veterinaria de la Universidad de la República, por sus aportes, sugerencias y orientación. Al Dr. Ralf Thiel, Ictiólogo Curador del Biocenter Grindel und Zoological Museum de la Universidad de Hamburgo por el aporte bibliográfico para la determinación del especímen, al Dr. Hebert Nion por el aporte de bibliografía y datos del ejemplar de Nemichthys scolopaceus capturado en Uruguay, al 
Tabla 1. Tamaño, caracteres merísticos y caracteres morfométricos (expresado en \% de SL100) de Labichthys yanoi (ejemplar MNHN 3867 y MNHN 3868). Ver también Figura 5.

\begin{tabular}{|c|c|c|}
\hline Ejemplar & MHNM 3867 & MHNM 3868 \\
\hline \multicolumn{2}{|l|}{ Tamaño } & \\
\hline Largo total (mm) & 395 & 670 \\
\hline SL100 (mm) & 363 & 460 \\
\hline Masa $(\mathrm{g})$ & 3,58 & 29,17 \\
\hline \multicolumn{2}{|l|}{ Caracteres merísticos } & \\
\hline Radios dorsales & 259 & 296 \\
\hline D100 & 139 & 135 \\
\hline Radios anales & 237 & 276 \\
\hline A100 & 115 & 125 \\
\hline Radios pectorales & 11 & 10 \\
\hline Poros en línea lateral (= vértebras) & 186 & 191 \\
\hline \multicolumn{2}{|l|}{ Caracteres morfométricos } & \\
\hline Diámetro del ojo & 0,96 & 1,08 \\
\hline Postorbital & 3,3 & 3,91 \\
\hline Preanal & 4,4 & 6,5 \\
\hline Predorsal & 5,6 & 4,34 \\
\hline
\end{tabular}

Dr. Daniel Figueroa, a la Dra. Berta Cosseau y al Dr. Juan Martín Díaz de Astarloa de la Universidad Nacional de Mar del Plata por los datos de nemíctidos en aguas argentinas, al Dr. Marcelo Melo de la Universidad de San Pablo por los datos de nemíctidos en aguas brasileras, al Sr. Fabrizio Scarabino (DINARA) por el aporte de bibliografía e información de Uruguay. Al Lic. Sebastián Serra (Museo Nacional de Historia Natural de Montevideo) por las correcciones al trabajo y la orientación en el trabajo de colección.

\section{REFERENCIAS}

Costa P.A.S., Braga A.C., Melo M.R.S., Nunan G.W.A., Martins A.S. \& Olavo G. 2007. Assembleias de teleósteos demersais no talude da costa central brasileira. En: P.A.S. Costa, G. Olavo \& A.S. Martins (Eds.) Biodiversidade da fauna marinha profunda na costa central brasileira, pp. 87107. Museu Nacional, Rio de Janeiro.

Cousseau M.B. 2010. Peces, Crustáceos y Moluscos registrados en el sector del Atlántico Sudoccidental comprendido entre $34^{\circ} \mathrm{S}$ y $54^{\circ} \mathrm{S}$, con indicación de las especies de interés pesquero, INIDEP Informe Técnico 5. 20 pp.

Cousseau M.B., Barbini S.A. \& Figueroa D.E. 2012. The presence of southern fishes in the
Argentinian continental shelf and adjacent areas. Marine Biodiversity, 42: 73-78.

Eastman J.T. 2005. The nature of the diversity of Antarctic fish. Polar Biology, 28: 93-107.

Figueroa D.E, Diaz De Astarloa J.M. \& Martos P. 1998. Mesopelagic fish distribution in the southwest Atlantic in relation to water masses. Deep-Sea Research, 45: 317-332.

Mead G.W. \& Rubbinoff I. 1966. Avocettinops yanoi, a new nemichthyid eel from the southern Indian Ocean. Breviora, 241: 1-6.

Melo M.R.S., Nunan G.W.A., Braga A.C. \& Costa P.A.S. 2009. The deep sea Anguilliformes and Saccopharingiformes (Teleostei: Elepomorpha) collected on the Brazilian continental slope, between $11^{\circ}$ y $23^{\circ} S$. Zootaxa, 2234: 1-20.

Menni R.C., Ringuelet R.A. \& Aramburu R.H. 1984. Peces marinos de la Argentina y Uruguay. Hemisferio Sur. Buenos Aires.

Nelson J. S. 2016. Fishes of the world, $5^{\text {th }}$ ed. John Wiley. New Jersey.

Nielsen J.G. \& Smith D.G. 1978. The eel Family Nemichthyidae (Pisces, Anguilliformes). DANA REPORT No. 88. Scandinavian Science Press. $71 \mathrm{pp}$.

Nion H., Ríos C. \& Meneses P. 2002. Peces del Uruguay: Lista sistemática y nombres comunes. Montevideo: Dirección Nacional de Recursos Acuáticos-Infopesca. 104 pp. 
Vaz Ferreira R., Ríos C. \& Nion H. 1999. Peces marinos nuevos o poco conocidos en Uruguay: Nota1. Boletín de la Sociedad Zoológica del Uruguay (2a Época), 10: 34-57.

Robins C. R. 1989. The phylogenetic relationships of the Anguilliform fishes. En: E.B. Böhlke (Ed.). Fishes of the Western North Atlantic. Memoirs of the Sears Foundation of Marine Research (№ 1, Part 9). pp 9-23. Yale University. New Haven.

Fecha de recepción: 10 de mayo de 2016 Fecha de aceptación: 18 de noviembre de 2016 